\title{
ALGEBRAS OF FOURIER TRANSFORMS WITH CLOSED RESTRICTIONS
}

BY

BENJAMIN B. WELLS, JR.

\begin{abstract}
Let $G$ denote a compact abelian group and let $B$ denote a Banach subalgebra of $A$, the algebra of complex-valued functions on $G$ whose Fourier series is absolutely convergent. If $B$ contains the constant functions, separates the points of $G$, and if the restriction algebra, $B(E)$, is closed in $A(E)$ for every closed subset $E$ of $G$, then $B=A$.
\end{abstract}

1. Introduction. Let $G$ denote a compact abelian group and $A$ the algebra under the pointwise operations of complex-valued functions on $G$ having absolutely convergent Fourier series. Thus, a function $f$ in $A$ has a representation as $\sum a_{n}\left(\gamma_{n}, g\right)$ where $\sum\left|a_{n}\right|<\infty$, the latter quantity serving as the norm of $f$.

Let $B$ denote a subalgebra continuously embedded in $A$, containing the constant functions, and separating the points of $G$. For $E$, a closed subset of $G, B(E)$ and $A(E)$ denote the restriction algebras to the set $E$ and are furnished with the quotient norm.

THEOREM. Suppose that for every closed set $E \subset G, B(E)$ is a closed linear subspace of $A(E)$. Then $B=A$.

This result is the exact analogue of a result [1] in function algebras with the role of the continuous complex-valued functions played by $A$. In his thesis [4] Sungwoo Suh proved the theorem for locally compact totally disconnected groups.

To illustrate the content of the Theorem, consider the case of $G$ equal to $T$, the circle group, and let $B$ be the closure of the linear span of $A$-functions taking the values 1 and 0 on a closed totally disconnected set $F \subset T$. It is known (see [2, p. 40], and [3]) that there are certain $F$ for which $B$ is a proper subalgebra of $A$. From our Theorem it follows that $B(E)$ fails to be closed in $A(E)$ for some closed $E$ that must intersect $F$ in a proper subset. A qualitative statement of this fact is the following. There are functions locally constant on $E$ that are the restrictions of $A$-functions having small $A$-norm, but such that any attempt to extend them to remain locally constant on $F$ will force their $A$-norms to become large.

We now indicate some notations and tools to be used in the proof of the Theorem. The norm of a function $f \in B(E)$ is given by

$$
\|f\|_{B(E)}=\inf \left\{\|f+h\|_{B}: h \in B \text { and } h=0 \text { on } E\right\} .
$$

If $f \in B(E)$, a representative of $f$ is a function $f_{0}$ belonging to $B$ such that $f_{0} \mid E=f$.

Received by the editors April 10, 1979 and, in revised form, December 10, 1979.

AMS (MOS) subject classifications (1970). Primary 43A25, 46E25.

() 1980 American Mathematical Society $0002-9947 / 80 / 0000-0371 / \$ 02.50$ 
The dual space of $A$ as a Banach space is $P M$, the space of pseudomeasures on $G$. The action of a pseudomeasure $S$ on an $A$-function $f$ will be denoted by $(S, f)$. The symbol $S f$ will denote that pseudomeasure such that $(S f, g)=(S, f g)$ for all $g \in A$. A pseudomeasure $S$ is said to be supported by $E$, and we write $S \in$ $P M(E)$, provided $(S, f)=0$ whenever $f$ vanishes in a neighborhood of $E$. The dual space of the restriction algebra $A(E)$ consists of all those pseudomeasures belonging to $P M(E)$ that annihilate every $A$-function vanishing on $E$. Finally, $B^{\perp}$ will denote all of those pseudomeasures that annihilate every function in $B$.

2. Preliminary lemmas. We begin by showing that under the assumptions of the Theorem the restriction algebra $B(E)$ must contain all of the idempotents of $A(E)$. In the function algebra case (cf. [1]) this step is almost immediate. In this section $G$ need not be a group, only a compact topological space. The algebra $A$ need only be a commutative semisimple regular Banach algebra.

Let $A$ be such an algebra under the pointwise operations on its maximal ideal space $G$, and let $B$ denote a closed subalgebra of $A$ containing 1 and separating the points of $G$.

LEMMA 1. Assume that for every closed subset $E \subset G$ the inclusion mapping $B(E) \rightarrow A(E)$ is closed. Then, for every closed subset $E \subset G, B(E)$ contains all the idempotent functions on $E$ belonging to $A(E)$.

Proof. Let $E$ be an arbitrary closed subset of $G$. We will show that the maximal ideal space of $B(E)$ is $E$. Thus, appealing to the Shilov idempotent theorem, we have that if $f$ belongs to $A(E)$ and is idempotent on $E$, then it must also belong to $B(E)$.

Let $h$ denote a complex homomorphism of $B(E)$, and suppose that $h$ is not evaluation at some point of $E$. Then for each $x \in E$, there exists a function $g$ belonging to $B(E)$ such that $h(g)=1$ and such that $g(x)=0$. Let $W_{x}$ be a closed neighborhood of $x$ such that $|g(y)| \leqslant 1 / 4$ for all $y \in W_{x}$. Of course, the maximal ideal space of $B\left(E \cap W_{x}\right), \Delta$, is a closed subset of the maximal ideal space of $B(E)$. We shall now show that $h$ does not belong to $\Delta$.

Suppose on the contrary that $h \in \Delta$, and define $a=\sup _{\phi \in \Delta}|\phi(g)|$. Then $a \geqslant 1$, and for every positive integer $n$

$$
\left\|(g / a)^{n}\right\|_{B\left(W_{x} \cap E\right)} \geqslant 1 .
$$

Since $|g(y)| \leqslant 1 / 4$ for all $y \in W_{x}$, and the maximal ideal space of $A\left(W_{x} \cap E\right)$ is just $W_{x} \cap E$, it follows that

$$
\lim \sup \left\|(g / a)^{n}\right\|_{A\left(W_{x} \cap E\right)^{1 / n}} \leqslant 1 / 4 .
$$

Thus, for $n$ large we have

$$
\left\|(g / a)^{n}\right\|_{A\left(W_{x} \cap E\right)} \leqslant(1 / 2)^{n} .
$$

Now, (1) and (2) together contradict the closedness of the mapping $B\left(W_{x} \cap E\right) \rightarrow$ $A\left(W_{x} \cap E\right)$. This establishes that $h$ does not belong to $\Delta$.

Therefore, there is a function $g \in B(E)$ that vanishes on $W_{x}$ such that $h(g)=1$. We may repeat the above argument for each point of $E$, and by the compactness of 
$E$ choose a finite set of functions $g_{i}, i=1, \ldots, n$, so that $h\left(g_{i}\right)=1$ and so that the product $g_{0}$ vanishes on $E$. Of course, $h\left(g_{0}\right)=1$. This contradicts the fact that $h$ was chosen in the maximal ideal space of $B(E)$. The proof of the lemma is complete.

Definition. Let $N_{E}$ denote the norm of the inverse of the inclusion mapping $B(E) \rightarrow A(E)$. Following [1], we say that $B$ is bounded on a subset $V$ of $G$ if there is a positive constant $C_{V}$ for which, whenever $F$ is closed and contained in $V$, $N_{F} \leqslant C_{V}$. We say that $B$ is bounded at $x \in G$ if $B$ is bounded on some neighborhood $V$ of $x$.

LeMma 2. Let $V_{1}$ and $V_{2}$ be open subsets of $G$ and let $B$ be bounded on each $V_{i}$, $i=1,2$. Then $B$ is bounded on every closed subset of $V_{1} \cup V_{2}$.

Proof. Choose a closed subset $F$ of $V_{1} \cup V_{2}$. Since $F \backslash V_{2}$ is a closed subset of $V_{1}$, we can find open $W_{1} \supset F \backslash V_{2}$ such that $\bar{W}_{1} \subset V_{1}$. Since $F \backslash W_{1} \subset V_{2}$, there is an open $W_{2} \supset F \backslash W_{1}$ such that $\bar{W}_{2} \subset V_{2}$. Now $F \subset W_{1} \cup W_{2}$. Furthermore, we may choose open $L$ satisfying $F \backslash W_{2} \subset L \subset \bar{L} \subset W_{1}$, and by Lemma 1 a function $h \in B$ that is 1 on $F \backslash W_{2}$ and 0 on $L^{c}$. For $f \in B(F)$ there are $f_{i} \in B, i=1,2$, such that $f_{i}\left|\bar{W}_{i}=f\right| \bar{W}_{i}$ and $\left\|f_{i}\right\|_{B}<C_{V_{i}}$. But now it is easy to check that $f=f_{1} \cdot h$ $+(1-h) f_{2}$ on $F$. We have, therefore

$$
\|f\|_{B(F)} \leqslant\left\|f_{1}\right\|_{B}\|h\|_{B}+\|1-h\|_{B}\left\|f_{2}\right\|_{B}<\|h\|_{B} C_{V_{1}}+\|1-h\|_{B} C_{V_{2}}
$$

The right-hand side of (3) may be taken to be $C_{F}$. The proof of the lemma is now complete.

Lemma 3. If $F$ is closed in $G$, and $B$ is bounded at each $x \in F$, there is an open $V$ containing $F$ on which $B$ is bounded.

Proof. The proof is an obvious consequence of compactness of $F$ and Lemma 2.

LEMMA 4. There are at most finitely many $x \in G$ at which $B$ is not bounded.

Proof. Assume on the contrary that $B$ is not bounded at each member of the sequence $\left\{y_{n}\right\}$. By dropping to a subsequence, if necessary, we may suppose that no element of the sequence $\left\{y_{n}\right\}$ is a limit point of the sequence. Choose open neighborhoods $U_{y_{1}}, W_{y_{1}}$ of $y_{1}$ that are disjoint from the set of limit points of the sequence $\left\{y_{n}\right\}$, such that $\bar{W}_{y_{1}} \subset U_{y_{1}}$. By Lemma 1 we may choose a function $k_{1} \in B$ that is 1 on $\bar{W}_{y_{1}}$ and 0 on $U_{y_{1}}^{c}$. Since $B$ is unbounded in every neighborhood of $y_{1}$, we may pick closed $F_{1} \subset W_{y^{\prime}}$ so that there is a $g_{1} \in B\left(F_{1}\right)$ with $\left\|g_{1}\right\|_{B\left(F_{1}\right)}>1$ while having $\left\|g_{1}\right\|_{A\left(F_{1}\right)}<\left\|k_{1}\right\|_{A}^{-1}$. Set $f_{1}=h_{1} k_{1}$ where $h_{1} \mid F_{1}=g_{1}$ and $h_{1}$ is a representative of $g_{1}$ whose $A$-norm is less than $\left\|k_{1}\right\|_{A}^{-1}$. Then $\left\|f_{1}\right\|_{A} \leqslant\left\|h_{1}\right\|_{A}\left\|k_{1}\right\|_{A}$ $<1$, but, of course, $\left\|f_{1}\right\|_{B\left(F_{1}\right)}>1$ since $f_{1}=g_{1}$ on $F_{1}$. Note that $f_{1}$ itself may not belong to $B$, since $h_{1}$ need not.

Next choose $y_{2}^{\prime} \in\left\{y_{n}\right\}_{n=2}^{\infty}$ and open neighborhoods $U_{y_{2}^{\prime}}, W_{y_{2}^{\prime}}$, of $y_{2}^{\prime}$ that are disjoint from the set of limit points of the sequence $\left\{y_{n}\right\}$, such that $\bar{W}_{y_{2}^{\prime}} \subset U_{y_{2}^{\prime}}$ and $U_{y_{1}} \cap U_{y_{2}^{\prime}}=\varnothing$. Choose a function $k_{2} \in B$ such that $k_{2}$ is 1 on $\bar{W}_{y_{2}^{\prime}}$ and is 0 on $U_{y_{2}^{\prime}}^{c}$. Since $B$ is not bounded in every neighborhood of $y_{2}^{\prime}$, it follows that there is a closed set $F_{2} \subset W_{y_{2}^{\prime}}$ and a function $g_{2} \in B\left(F_{2}\right)$ with $\left\|g_{2}\right\|_{B\left(F_{2}\right)}>2$, but $\left\|g_{2}\right\|_{A\left(F_{2}\right)}<\left\|k_{2}\right\|_{A}^{-1}$. 
Set $f_{2}=h_{2} \cdot k_{2}$ where $h_{2} \mid F_{2}=g_{2}$ and $h_{2}$ is a representative of $g_{2}$ whose $A$-norm is less than $\left\|k_{2}\right\|_{A}^{-1}$. Then $\left\|f_{2}\right\|_{A}<1$, but, of course, $\left\|f_{2}\right\|_{B\left(F_{2}\right)}>2$. Continuing in this way, we arrive at a disjoint sequence of closed sets $\left\{F_{n}\right\}$ and a sequence of functions $\left\{f_{n}\right\}$ such that

$$
\left\|f_{n}\right\|_{A}<1, \quad\left\|f_{n}\right\|_{B\left(F_{n}\right)}>n, \quad n=1,2, \ldots
$$

Denote by $F$ the closed set $\cup_{n=1}^{\infty} F_{n} \cup F_{0}$, where $F_{0}$ is the set of limit points of $\cup_{n=1}^{\infty} F_{n}$. For each $m, f_{m} \mid F_{m} \in B\left(F_{m}\right)$ and $f_{m}=0$ on the closed set $\cup_{n \neq m} F_{n} \cup$ $F_{0}$. The latter set is disjoint from $F_{m}$. It follows from Lemma 1, by multiplying any representative of $f_{m} \mid F_{m}$ in $B$ by a function in $B$ equal to 1 on $F_{m}$ and 0 on $\cup_{n \neq m} F_{n} \cup F_{0}$, that $f_{m} \in B(F)$. Therefore, the closedness of the inclusion mapping $B(F) \rightarrow A(F)$ is contradicted by (4). The proof of the lemma is complete.

3. Proof of the Theorem for the circle $T$. Assume on the contrary that $B \neq A$ and that $H$ is the finite set of points at which $B$ is not bounded. Our first claim is that there is a closed set $F$ such that $F^{c}$ contains $H$ and such that $B(F) \neq A(F)$. If not, then for every such $F, B(F)=A(F)$. Let $S$ denote a pseudomeasure in $B^{\perp}$, and suppose that $f$ belongs to $A$ and is 0 in a neighborhood of $H$. It follows from Lemma 1 that $B$ is a normal algebra of functions on $T$. Therefore, since $f$ belongs locally to $B$ at each point of $T, f$ belongs to $B$. Since $S$ is orthogonal to $B$, $(S, f)=0$. Thus the support of $S$ is the finite set $H$, and $S$ is in fact a measure. Since $B$ separates the points of $T$, it follows that $S\{p\}=0$ for every $p \in H$. This establishes that $B(F) \neq A(F)$ for some closed set $F$ such that $F^{c}$ contains $H$. Henceforth we shall assume that $F^{c}$ is a finite union of disjoint open intervals centered at the points of $H$. Then, by Lemma $4, B$ is bounded, say with constant $N_{W}$, on some open set $W$ containing $F$ and missing $H$.

Now, at least one of the functions $e^{ \pm i x}$, say $e^{i x}$, when restricted to $F$, fails to belong to $B(F)$. By the Hahn-Banach Theorem there is a nonzero element $S$ of $A(F)^{*}$ that annihilates $B(F)$ and such that $\left(S, e^{i x}\right)=1$. We shall regard $S$ as a pseudomeasure supported on $F$.

Let $m$ be a fixed positive integer satisfying

$$
\exp (20 / m)<1+\left(8 e^{20}\|S\| N_{W}^{2} C\right)^{-1},
$$

where $C$ denotes the norm of the mapping $B \rightarrow A$.

Let $\sigma$ denote the trapezoidal function equal to 1 on the interval $(-\pi / 4, \pi / 4)$, zero outside the interval $(-\pi / 2, \pi / 2)$ and linear on intermediate intervals. Set $V_{1}(x)=\sigma(m x)$ and $V_{2}=1-V_{1}$. From standard estimates it follows that $\left\|V_{j}\right\|_{A} \leqslant$ 4 for $j=1,2$. By Lemma 1 and the boundedness of $B$ on $W$, there is a $B$-function $v_{1}$ with its $B$-norm bounded by $4 N_{W}$, and such that $v_{1}=V_{1}$ on the intersection of $F$ with the intervals where $V_{1}$ is constant. Set $v_{2}=1-v_{1}$.

Since $\left(S, e^{i x}\right)=1$, it follows that for at least one $j,\left|\left(S v_{j}, e^{i x}\right)\right| \geqslant 1 / 2$. There will be no loss of generality in assuming that this occurs for $j=1$. The ensuing argument is similar in the other case. Let $K$ denote the support of the function $v_{1}$. Of course, since $S$ is orthogonal to $B$, so also is the pseudomeasure $L=S v_{1}$. The latter's support is contained in the finite union of closed intervals $F \cap K$, and its 
pseudomeasure norm satisfies

$$
\|L\| \leqslant\|S\|\left\|v_{1}\right\|_{A} \leqslant 4\|S\| N_{W} C .
$$

Let $\zeta(x)$ denote the continuous piecewise linear function such that $\zeta(-\pi / 2)=0$, $\zeta(\pi / 2)=\pi$ and $\zeta$ has slope -1 on the intervals complementary to $(-\pi / 2, \pi / 2)$. Set $Z(x)=\zeta(m x)$. By standard estimates the $A$-norm of $Z$ is no greater than 20 . The important property for present purposes of the function $Z$ is that the restriction of the function $e^{i Z / m}$ to the support of $v_{1}$ is just $\exp \left\{i\left(x+\lambda_{j}\right)\right\}$ for constants $\lambda_{j}$ and $x$ belonging to the $j$ th interval of support of $v_{1}, j=1,2, \ldots, m$.

The $A$-norm of the function $e^{i(x-z / m)}$ is no greater than $e^{20}$. On the set $F \cap K$ it is equal to the constant $e^{-i \lambda}$ for $x$ belonging to the $j$ th interval of $K$. In particular, it is locally constant on $F \cap K$ and so belongs to $B(F \cap K)$. Its $B(F \cap K)$-norm is no greater than $N_{W} e^{20}$. Let $\alpha(x)$ be a representative in $B$ of the restriction of $e^{i(x-Z / m)}$ to the set $F \cap K$ satisfying

$$
\|\alpha\|_{B} \leqslant N_{W} e^{20}
$$

Likewise, let $\alpha_{1}(x)$ be a representative in $B$ of $e^{-i(x-z / m)}$ restricted to $F \cap K$. Thus, we have that

$$
1 / 2 \leqslant\left|\left(L \alpha \alpha_{1}, e^{i x}\right)\right|=\left|\left(L \alpha, \alpha_{1} e^{i x}\right)\right|=\left|\left(L \alpha, e^{i Z / m}\right)\right| .
$$

Since $\alpha \in B$, the pseudomeasure $L \alpha$ is still orthogonal to $B$.

We have

$$
\left\|e^{i Z / m}-1\right\|_{A(F \cap K)} \leqslant \exp \left\{\|Z\|_{A} / m\right\}-1 .
$$

From the triangle inequality we see that

$$
\left|\left(L \alpha, e^{i Z / m}\right)\right| \leqslant\left|\left(L \alpha, e^{i Z / m}-1\right)\right|+|(L \alpha, 1)| .
$$

Since $L \alpha$ is orthogonal to $B$, the second term on the right-hand side of $(10)$ is 0 . Since the set $F \cap K$ is a finite union of closed intervals, $L \alpha$ may be regarded as an element of the dual space of $A(F \cap K)$. Therefore, the first term on the right-hand side of (10) is at most $\|L\|\|\alpha\|_{A}\left\|e^{i Z / m}-1\right\|_{A(F \cap K)}$. By our choice of $m$ in (5) and by inequalities (6), (7) and (9), it follows that the right-hand side of (10) is less than $1 / 2$. This contradicts (8) and completes the proof of the Theorem for the case of the circle.

4. Proof of the Theorem for an arbitrary compact abelian group. First we consider a product of circles $\Pi_{\alpha} T_{\alpha}$. Let $f$ denote the element in the dual group constant in every coordinate except the $\beta$ th coordinate, and of the form $e^{i x}$ in the $\beta$ th coordinate. Consider the products $E=\Pi_{\alpha \neq \beta} T_{\alpha} \times E_{\beta}$, where the projection onto the $\beta$ th coordinate is a closed set $E_{\beta}$.

Since $B(F)$ is closed in $A(F)$ for all $F$ closed and contained in $\Pi_{\alpha} T_{\alpha}$, it follows from Lemma 1 that $B(E)$ contains the span of the idempotents of $A(E)$. By Lemma $4 B$ is bounded on the complement of any neighborhood of a certain finite set of points. To deny that $f$ belongs to $B$ leads to a contradiction as before. Therefore, all characters having the form of $f$ belong to $B$, and since $B$ is closed in $A$ it must equal $A$. 
Finally, let $G$ be an arbitrary compact abelian group. Then every neighborhood of the identity contains a closed subgroup $H$ such that $G / H$ is isomorphic to a (finite) product of circles with a finite group. By the above remarks and Lemma 1 , $B$ will contain those characters of the dual group $G / H$. If $B(G / H)$ is interpreted to mean those $B$-functions constant on the cosets of $H$, it follows that $B(G / H)=$ $A(G / H)$. In particular, $B$ contains all trigonometric polynomials, and by the closedness of $B$ in $A$, we again conclude that $B=A$.

\section{REFERENCES}

1. I. Glicksberg, Function algebras with closed restrictions, Proc. Amer. Math. Soc. 14 (1963), 158-161.

2. J.-P. Kahane, Séries de Fourier absolument convergentes, Ergebnisse der Math. und ihrer Grenzgebiete, Band 50, Springer-Verlag, Berlin and New York, 1970.

3. Y. Katznelson and W. Rudin, The Stone-Weierstrass property in Banach algebras, Pacific J. Math. 11 (1961), 253-265.

4. Sungwoo Suh, Characterization of $L^{\prime}(G)$ among its subalgebras, Thesis, Univ. of Connecticut, 1978.

Department of Mathematics, Universtty of Hawail at Manoa, Honolulu, Hawail 96822 